\title{
CAD Modeling of Coplanar Waveguide Cross-Over Air-Bridge
}

\author{
Nihad Dib \\ Electrical Engineering Dept., Jordan University of Science and Technology, P.O. Box 3030, Irbid \\ 22110, Jordan
}

Received 6 September 2004; accepted 24 October 2004

\begin{abstract}
In this article, a new CAD model for the coplanar waveguide (CPW) cross-over air-bridge is proposed. The model takes into account the CPW open-end capacitance and the vertical strips of the bridge. The section directly underneath the bridge is modeled as a small section of a microstrip line. Our results are in good agreement with the published full-wave results. (C) 2005 Wiley Periodicals, Inc. Int J RF and Microwave CAE 15: 251-254, 2005.
\end{abstract}

Keywords: coplanar waveguide; air-bridge; CAD

\section{INTRODUCTION}

Uniplanar circuits for MMICs, in which only one side of the substrate is used, have been proposed in [1]. These circuits use a combination of coplanar waveguide (CPW) and slotline. One of the key components for the uniplanar structure is the air-bridge, which is used to connect the CPW to slotlines or coupledslotlines. Air-bridges may have parasitic problems when the sizes involved are large or if their number is large [1].

In this article, the CPW cross-over air-bridge is studied, as shown in Figure 1 where $W$ is the slot width, $S$ is the center conductor width, $h$ is the substrate thickness with a dielectric constant $\varepsilon_{r}, d$ is the length of the air-bridge, $h_{a}$ is the height of the airbridge, and $g$ is the open-end gap width. In this article, the metallization is assumed to be a perfect conductor with zero thickness.

In [1], this CPW bridge was modeled as an airfilled microstrip line along with fringing capacitances, and it was shown that there is no discontinuity prob-

Correspondence to: N. Dib; email: nihad@just.edu.jo.

DOI 10.1002/mmce.20075

Published online 24 January 2005 in Wiley InterScience (www. interscience.wiley.com). lem if the size of the bridge is small. Full-wave TLM and FDFD methods were used to study this bridge in [2-4], where it was found that such a bridge could behave as an inductive or capacitive element, depending on the bridge height and length [2]. Moreover, the same bridge has been experimentally studied in [5].

In this article, a new equivalent-circuit model for the CPW cross-over air-bridge is introduced and investigated. The results are compared to published full-wave results and a good agreement is obtained.

\section{EQUIVALENT-CIRCUIT MODEL}

Referring to Figure 1, it can be seen that the cross section in the crossing region may be regarded as an air-filled microstip line of substrate height $h_{a}$, and strip width $S$. In the open-end gap region, the cross section may be regarded as an elevated strip CPW with center conductor width $S$, and gap width $W$.

Figure 2 shows a cross section of the elevated strip CPW (ESCPW). Recently, the ESCPW has been the subject of many papers in the literature [6-12]. The ESCPW has some advantages over the conventional $\mathrm{CPW}$. It has fewer losses and covers a broader range of characteristic impedances. The ESCPW has been mainly analyzed using the full-wave FDTD method 


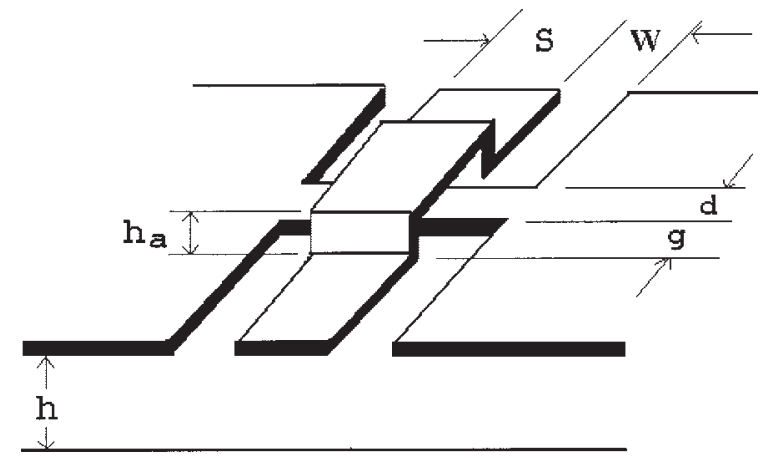

Figure 1. CPW cross-over air-bridge.

$[6,7]$, the quasi-static finite-difference technique [8], and the finite-element method [9]. To our knowledge, no closed-form expressions for the effective dielectric constant and the characteristic impedance of the ES$\mathrm{CPW}$ are available. Based on conformal-mapping analysis of the recessed strip CPW in [13], we tried to use conformal mapping to obtain such expressions, but found out that this could be tedious and not really necessary for our air-bridge study, as will be shown below.

Given the above discussion, Figure 3 shows the proposed equivalent-circuit model for the air-bridge. The transmission line of length $d$ is the air-filled microstrip line, while those of lengths $g$ are the ES$\mathrm{CPW}$ sections. The capacitance $C_{o e}$ is the CPW openend capacitance, which was evaluated using expressions from $[14,15]$. The inductance $L_{v s}$ is the series inductance due to the vertical strip of the air bridge connecting the center strips of the CPW and the ESCPW. This inductance can be approximated using the following expression [16]:

$$
L_{v s}=\frac{\mu_{0} h_{a}}{2 \pi}\left[\ln \left(\frac{1+\sqrt{1+p^{2}}}{p}\right)+p-\sqrt{1+p^{2}}\right]
$$

where $p=S /\left(4 h_{a}\right)$.

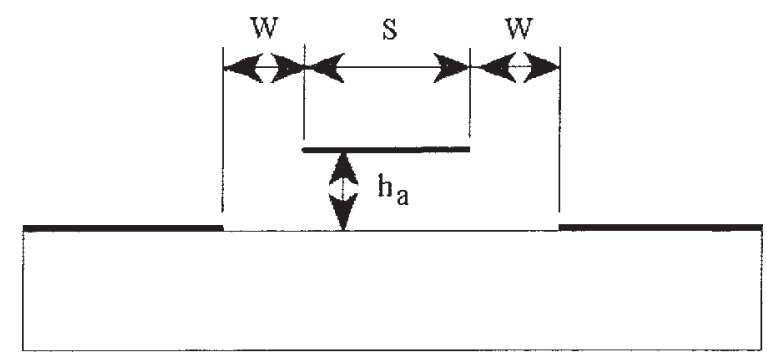

Figure 2. Cross section of an elevated strip coplanar waveguide (ESCPW).

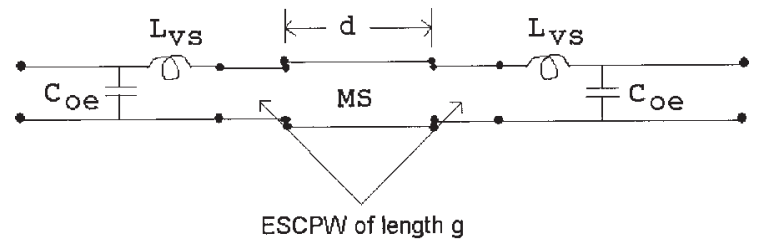

Figure 3. Equivalent-circuit model for the CPW airbridge.

As mentioned above, a closed-form expression for the impedance and effective dielectric constant of the ESCPW are not currently available; thus, it was decided to replace the very short ESCPW sections with equivalent lumped inductances. Figure 4 shows the resultant equivalent-circuit model, in which $L_{a}$ is the total inductance. After extensive numerical experiments and comparing the obtained results to those from [2, 3], the following expression was used to evaluate $L_{a}$ :

$$
\begin{gathered}
L_{a}=L_{1}+L_{2}, \\
L_{1}=\frac{\mu_{0}\left(h_{a}+g\right)}{2 \pi}\left[\ln \left(\frac{1+\sqrt{1+p^{2}}}{p}\right)+p-\sqrt{1+p^{2}}\right], \\
L_{2}=(g)\left(L_{E S C P W}\right), \\
L_{E S C P W}=L_{C P W}=\sqrt{\mu_{0} \varepsilon_{0} \varepsilon_{\text {effCPW }}} Z_{0 C P W},
\end{gathered}
$$

where $p=S / 4\left(h_{a}+g\right)$. In the above equations, $L_{1}$ is the inductance of a flat wire of length $h_{a}+g$ and width $S$, while $L_{2}$ is the inductance of the ESCPW sections. It should be noted that the inductance per unit length of the ESCPW is assumed to be equal to that of the conventional $\mathrm{CPW}$, as found in $[6,7]$. Finally, from this equivalent circuit, one can easily find the scattering parameters.

\section{RESULTS}

The CPW air-bridge under consideration has the following dimensions: $h=100 \mu \mathrm{m}, \varepsilon_{r}=12.9, S=15$ $\mu \mathrm{m}, W=10 \mu \mathrm{m}, h_{a}=3 \mu \mathrm{m}, d=30 \mu \mathrm{m}$, and $g=$

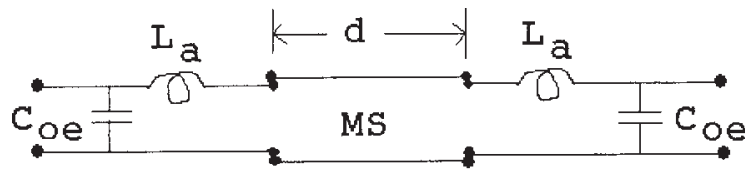

Figure 4. Final equivalent-circuit model for the CPW air-bridge. 


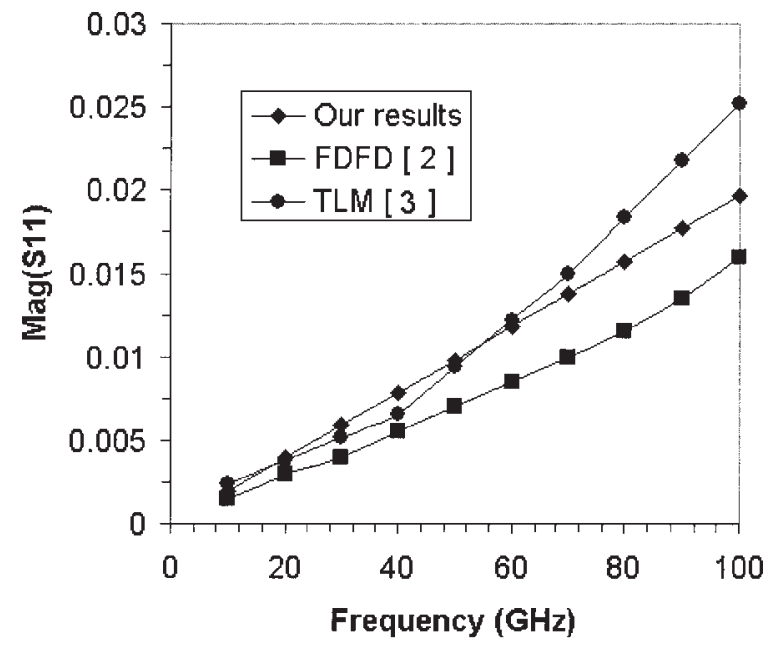

Figure 5. Magnitude of $S_{11}$ for the CPW air-bridge with the following dimensions: $h=100 \mu \mathrm{m}, \varepsilon_{r}=12.9, S=15$ $\mu \mathrm{m}, W=10 \mu \mathrm{m}, h_{a}=3 \mu \mathrm{m}, d=30 \mu \mathrm{m}$ and $g=7.5 \mu \mathrm{m}$.

$7.5 \mu \mathrm{m}$. These dimensions correspond to the airbridge analyzed in $[2,3]$. Using the equivalent circuit presented in the previous section, the obtained results are shown in Figures 5 and 6, along with full-wave results from $[2,3]$. It can be seen that our results are in good agreement with the full-wave FDFD and TLM results. From Figure 6, it can be concluded that the bridge under consideration behaves as an inductive element.

As mentioned in the Introduction, the CPW crossover bridge can behave as an inductive or capacitive element, depending on the bridge height $h_{a}$ and length $d$. Figure 7 shows our results for the phase of $S_{11}$ for a bridge height $h_{a}=2 \mu \mathrm{m}$ and two different lengths $d=23 \mu \mathrm{m}$ and $d=24 \mu \mathrm{m}$. It can be seen that for

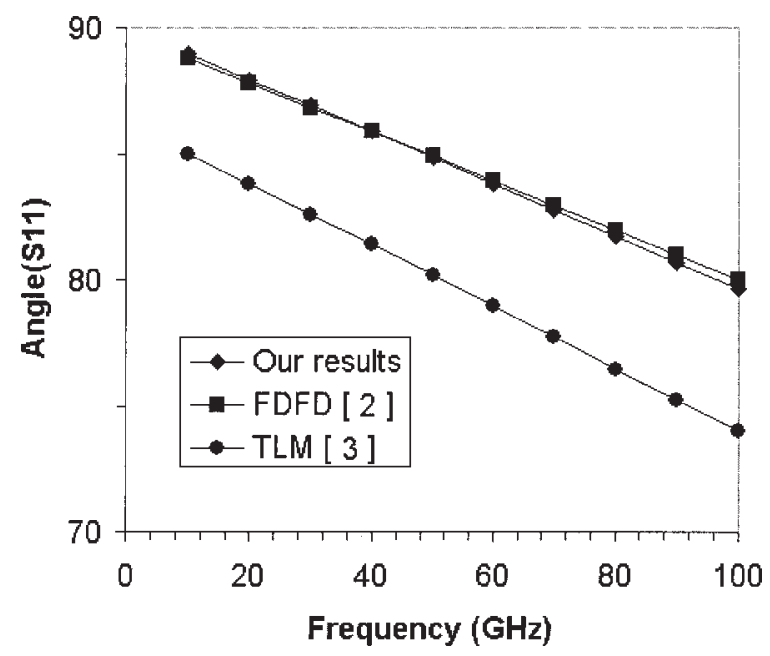

Figure 6. Phase of $S_{11}$ for the CPW air-bridge.

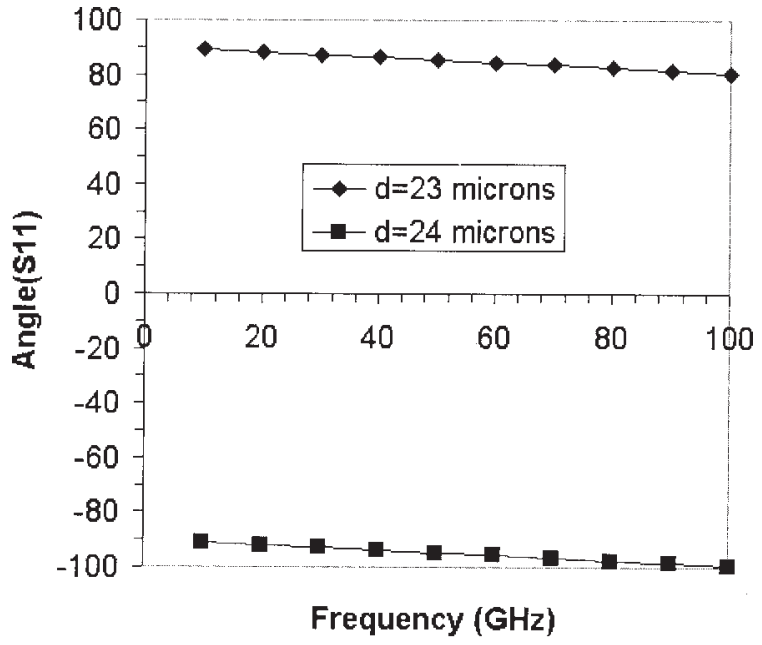

Figure 7. Phase of $S_{11}$ for the CPW air-bridge with $h_{a}=$ $2 \mu \mathrm{m}$ and two different lengths. $h=100 \mu \mathrm{m}, \varepsilon_{r}=12.9$, $S=15 \mu \mathrm{m}, W=10 \mu \mathrm{m}$, and $g=7.5 \mu \mathrm{m}$.

lengths less than $24 \mu \mathrm{m}$, the bridge behaves as an inductive element, while it behaves as a capacitive element for lengths larger or equal to $24 \mu \mathrm{m}$. This agrees well with an observation in [2], where it was found that for $h_{a}=2 \mu \mathrm{m}$, the bridge under consideration behaves as an inductive element for lengths $d<20 \mu \mathrm{m}$, and as a capacitive element for lengths $d>20 \mu \mathrm{m}$ (at $50 \mathrm{GHz}$ ). One can refer to [2] for an explanation of such a behavior.

\section{CONCLUSION}

From the above results, it should be noted that the studied CPW bridge problem is by no means a trivial one for any full-wave method, since the bridge size is very small, compared to a wavelength. All full-wave techniques have method-related parameters (for example, mesh size) that certainly give some inherent errors in each technique, especially when the magnitude of $S_{11}$ is in the order of -40 to $-60 \mathrm{~dB}$. Thus, it is not really justified to use computationally and/or analytically intensive full-wave methods to obtain the small parasitic effects of a single air-bridge. Instead, for all practical purposes, the simple circuit model presented in this article can be used to model the CPW cross-over air-bridge. This circuit model consists of a small section of an air-filled microstrip line along with a shunt capacitance and series inductor. The results from such a model were compared to published fullwave analysis results and were found to be acceptable in a wide frequency range. It is rather difficult to obtain a range of validity for the presented expres- 
sions; however, since the circuit model is based on physical modeling of the bridge, it is believed that the model is valid for typical CPW cross-over air-bridges used in MMICs.

\section{REFERENCES}

1. T. Hirota, Y. Tarusawa, and H. Ogawa, Uniplanar MMIC hybrids: A proposed new MMIC structure, IEEE Trans Microwave Theory Tech 35 (1987), 576581.

2. K. Beilenhoff, W. Heinrich, and H. Hartnagel, The scattering behavior of air-bridges in coplanar MMICs, Proc Euro Microwave Conf, Stuttgart, 1991, pp. 11311135.

3. H. Jin and R. Vahldieck, Calculation of frequency-dependent $S$-parameters of CPW air-bridges considering finite metallization thickness and conductivity, IEEE MTT-S Int Microwave Symp Dig 1 (1992), 207-210.

4. H. Jin and R. Vahldieck, Full-wave analysis of CPW discontinuities using the frequency domain TLM method, IEEE Trans Microwave Theory Tech 41 (1993), 1538-1542.

5. N. Koster, S. Kosslowski, R. Bertenburg, S. Heinen, and I. Wolff, Investigations on air-bridges used for MMICs in CPW technique, Proc Euro Microwave Conf, London, 1989, pp. 666-671.

6. S. Hofschen, and I. Wolff, Simulation of an Elevated CPW Using the 2-D FDTD, IEEE Microwave Guided Wave Lett 6 (1996), 28-30.

7. A. Reichelt, and I. Wolff, New coplanar-like transmission lines for application in monolithic integrated millimeter-wave and submillimeter-wave circuits, IEEE MTT-S Int Microwave Symp Dig 1 (1998), 99-102.
8. H. Kamitsuna, A very small, low-loss MMIC rat-race hybrid using elevated CPW, IEEE Microwave Guided Wave Lett 6 (1992), 337-339.

9. S. Raju, B. Nityanandan, and V. Abhaikumar, Analysis of a shielded elevated coupled CPW using a modified finite-element method, Int J Microwave MillimeterWave CAE 6 (1996), 305-318.

10. U. Bhattacharya, S. Allen, and M. Rodwell, DC-725$\mathrm{GHz}$ sampling circuits and subpicosecond nonlinear transmission lines using elevated CPW, IEEE Microwave Guided Wave Lett 5 (1995), 50-52.

11. H. Kim, S. Jung, J. Park, C. Baek, Y. Kim, and Y. Kwon, A new micromachined overlay CPW structure with low attenuation over wide impedance ranges and its application to low-pass filters, IEEE Trans Microwave Theory Tech 49 (2001), 1634-1639.

12. B. Agarwal, A. Schmitz, J. Brown, M. Matloubian, M. Case, M. Le, M. Lui, and M. Rodwell, 112-GHz, $157-$ $\mathrm{GHz}$, and 180-GHz InP HEMT traveling-wave amplifiers, IEEE Trans Microwave Theory Tech 46 (1998), 2553-2559.

13. Y. Wang, and J. Okoro, Impedance calculations for modified coplanar waveguides, Int $\mathrm{J}$ Electron 68 (1990), 861-875.

14. K. Beilenhoff, H. Klingbeil, W. Heinrich, and H. Hartnagel, Open and short circuits in coplanar MMICs, IEEE Trans Microwave Theory Tech 41 (1993), 15341537.

15. M.H. Mao, R.B. Wu, C.H. Chen, and C.H. Lin, Characterization of CPW open end capacitance: Theory and experiment, IEEE Trans Microwave Theory Tech 42 (1994), 1016-1024.

16. W. Getsinger, Circuit duals on planar transmission media, IEEE MTT-S Int Microwave Symp Dig 1 (1983), $154-156$.

\section{BIOGRAPHY}

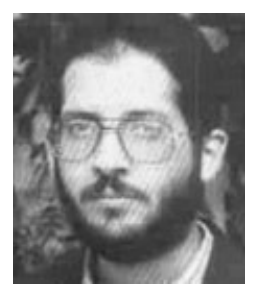

Nihad Dib received B.Sc. and M.Sc. degress in electrical engineering from Kuwait University in 1985 and 1987, respectively. He received his Ph.D. in electrical engineering (majoring in electromagnetics and microwaves) in 1992 from the University of Michigan, Ann Arbor. He worked as an Assistant Research Scientist in the Radiation Laboratory at the same school. In Septemeber 1995, he joined the Electrical Engineering Department at Jordan University of Science and Technology (JUST) as an Assistant Professor, and became an Associate Professor in September 2000. His research interests are in computational electromagnetics and modeling of planar circuits. 